Apidologie, 1981, 12 (4), 303-317.

\title{
COELIOXYS RUFOCAUDATA SM. (HYMENOPTERA MEGACHILIDAE) CLEPTOPARASITE DE MEGACHILE ROTUNDATA F. (HYMENOPTERA MEGACHILIDAE) POLLINISATEUR DE LA LUZERNE
}

\author{
S. CARRE et J.-P. PY \\ Laboratoire de Zoologie. I.N.R.A., 86600 Lusignan
}

\section{RÉSUMÉ}

Le développement en France des élevages expérimentaux de Megachile rotundata pour la pollinisation de la luzerne a conduit les auteurs à étudier la biologie des ennemis naturels de cette abeille en vue de leur élimination.

n 1980, l'espèce Coelioxys rufocaudata représente $88 \%$ des pertes de cocons dues aux parasites. De 1975 à 1980 le pourcentage des cellules parasitées par Coelioxys est passé au moins de $1 \%$ à $30,6 \%$ avec une multiplication voisine de 3 pendant 2 années consécutives. Une même cellule peut recevoir de 1 à 4 œufs en conditions d'élevage en champ. Une épaisseur minimum de 2 à $3 \mathrm{~mm}$ de pollen est nécessaire pour déclencher la ponte. Celle-ci ne se produit plus en fin d'approvisionnement de la cellule. Le développement comporte cinq stades larvaires. Au cours du troisième stade la larve de Coelioxys atteint la surface de la provision et détruit l'œuf ou la jeune larve de l'hôte au moyen de fortes mandibules. Ces dernières sont plus développées chez la femelle. Le quatrième et le cinquième stade ont une morphologie et un comportement alimentaire plus typiques des Megachilidae. La diapause s'effectue comme celle de l'hôte au stade prénymphal. Le taux de femelles dans la pollution varie de $28 \%$ à $49 \%$. Le léger décalage des émergences entre les deux espèces et le comportement de sommeil seront étudiés dans la perspective d'une méthode de lutte.

\section{INTRODUCTION}

Les conditions particulières de l'élevage de masse de l'abeille solitaire Megachile rotundata $\mathrm{F}$. ont favorisé l'extension rapide d'ennemis naturels. Coelioxys rufocaudata $\mathrm{Sm}$. est actuellement le parasite le plus menaçant pour l'avenir des élevages de Mégachiles en France. Les Coelioxys ou " abeilles coucou " sont des mégachilidés cleptoparasites dépourvus d'organes de récole du pollen. On en rencontre 17 espèces en France. Ils s'attaquent principalement au genre Megachile (BOHART, 1970) et secondairement aux Anthophorides du genre Anthophora (Perez, 1889; Friese, 1923; Richards, 
1949; Rozen, 1969). Ces Apoïdes sont caractérisés par un abdomen conique prolongé chez la femelle par un apex effilé. Pendant l'absence de l'abeille occupée au butinage, la femelle de Coelioxys pénètre dans le nid de l'hôte, perce la provision de pollen ainsi que les feuilles de la base de la cellule et dépose son œuf à travers la fente; seule la partie antérieure de l'œuf baigne dans la provision. C'est le cas de Coelioxys quadridentata $\mathrm{L}$. (Ferton, 1896), C. elongata LeP. (IwATA, 1939), C. octodentata Say (Michener, 1953 a). Ce mode de ponte se rencontre aussi chez des Nomadinae parasites : Holcopasites (Rozen, 1965), Triepeolus (BoHART, 1966), Pseudodichroa (Rozen, 1968). Cependant on signale d'autres modes de ponte : la femelle dépose son œuf à côté de l'œuf de l'hôte chez C. afra Lep. (Ferton, 1896) C. acuminata Nyl (Hardouin, 1948), C. decipiens Spin. (WAFA, 1973) ou suspend son auf au plafond de la cellule chez C. rufescens LEP. (FRIESE, 1923).

Nous nous proposons dans cette première note d'examiner les modalités d'infestation des nids de $M$. rotundata par $C$. rufocaudata dans les conditions d'élevage en champ. La ponte et le développement larvaire seront également étudiés sous l'aspect morphologique et comportemental.

\section{MATÉRIEL ET MÉTHODES}

Les résultats acquis proviennent de 3 types d'élevage :

1. Des élevages expérimentaux de $M$. rotundata sur luzernière dans le Sud de la France. Ces essais ont été réalisés avec l'aide de la F.N.A.M.S. (1) en 1975 dans les Alpes de Haute-Provence, l'Aude, la Haute-Garonne, le Tarn et le Vaucluse, et de 1976 à 1980 dans l'Aude, le Gers, la Drôme et le Tarn.

2. D'élevages contrôlés de l'hôte et de son parasite à Lusignan (1980).

- sur luzerne, en conditions d'élevage.

Un abri en polyane avec nichoir en bois (TASEI, 1975) est installé fin juin sur une pépinière de luzerne. Une population de 2500 cocons issue des essais F.N.A.M.S. 1980 et comportant $20,6 \%$ de Coelioxys est placée en éclosoir après réactivation à $30^{\circ} \mathrm{C}$ pendant 3 semaines. On suit quotidiennement l'évolution de la nidification et on effectue des observations sur le comportement du parasite adulte. Après retrait du nichoir fin septembre, on examine et on isole chaque cellule afin de repérer les pontes et les capsules céphaliques rejetées à l'extérieur des cocons tissés par les larves parasites. Les cocons sont ensuite mis en hibernation à $5^{\circ} \mathrm{C}$ et réactivés au bout de cinq mois afin d'identifier avec certitude l'occupant de chaque cellule.

- en compartiment de serre.

Après émergence on place 20 mâles et 20 femelles de Coelioxys avec autant d'individus de M. rotundata F. pour un compartiment de serre de $20 \mathrm{~m}^{3}$ environ. Cinquante caissettes de mélilot blanc pourvoient aux besoins des abeilles et de leur parasite. Trois pièges nichoirs constitués de tubes de bambou (TASEi, 1972) d'un diamètre de $5 \mathrm{~mm}$ sertis dans un bloc de bois permettent de contrôler à tout moment l'évolution de chaque nid. Il suffit de séparer les deux parties du tube fendu longitudinalement après retrait de l'élastique qui les maintient réunies.

(1) Fédération nationale des agriculteurs multiplicateurs de semences. 
3. D'élevage de larves de Coelioxys en conditions contrôlées.

La simple observation de la cellule n'est pas toujours suffisante pour détecter la présence des œufs de Coelioxys. Une ouverture minutieuse du cocon sous la loupe binoculaire peut être nécessaire afin de retirer les enveloppes de feuilles les plus externes. Les cellules parasitées sont ensuite maintenues verticalement dans des boîtes de polystyrène grillagées et placées en étuve à $24^{\circ} \pm 1^{\circ} \mathrm{C}$ et $60 \%$ d'humidité relative. Les larves destinées à l'étude morphologique sont extraites avec précaution de la provision, lavées dans l'alcool au pinceau fin et conservées dans l'alcool à $70^{\circ}$ avant d'être montées dans du liquide de Berlèse.

\section{RÉSULTATS}

1. Évolution annuelle (Sud de la France 1975 à 1980) et saisonnière (Lusignan 1980) du parasitisme de Coelioxys rufocaudata dans les élevages de Megachile rotundata.

- La souche de mégachiles importée du Canada en 1975 et utilisée pour les essais de 1975 à 1980 était exempte de C. rufocaudata. Le tableau 1 permet de suivre la progression du parasite pendant six années consécutives. La contamination s'est donc effectuée sur les champs d'essais dès la première année d'expérimentation où l'on relevait leur présence dans trois localités sur onze. En l'absence de techniques de lutte, $C$. rufocaudata prend une extension considérable à partir de 1978 où il occasionne près de $80 \%$ des pertes globales dues aux ennemis naturels. Leur coefficient de multiplication voisin de 3 pour 1978 et 1979 est égal à 1,5 en 1980. En 1980, le taux de femelles de $C$. rufocaudata varie de 33,6 à $48,8 \%$ et celui de son hôte de 28,2 à $35,5 \%$. Le pourcentage de cellules parasitées se situe entre $25 \%$ et $41 \%$ suivant les lieux d'élevage.

- La figure 1 montre le nombre de cocons parasités par C. rufocaudata en fonction de la date de construction des cellules. L'élevage a été mis en place le $1^{\text {er }}$ juillet, les premières femelles de Coelioxys sont entrées en activité le 17 juillet, la dernière femelle a été observée le 22 septembre. La baisse d'activité des mégachiles au mois d'août s'explique par des conditions climatiques très médiocres. Dans cet essai le pourcentage de cellules parasitées atteint $44,5 \%$. Près de $28 \%$ des Coelioxys qui en émergent sont des femelles.

\section{La phase imaginale.}

- Activité des adultes et comportement de ponte.

Le seuil d'activité des Coelioxys est plus élevé par rapport à son hôte. Dans les conditions climatiques du mois de juillet 1980 à Lusignan on a observé une durée de vol moyenne de 6 heures contre 8 heures pour $M$. rotundata. Les femelles de Coelioxys ne volent pas au-dessous de $21^{\circ} \mathrm{C}$. Au-delà, l'envol est très lié à l'ensoleillement. Pendant les phases d'arrêt de l'activité diurne liées à un abaissement de température ou d'ensoleillement et distinctes des périodes de repos courtes, les Coelioxys prennent la position de sommeil (Photo 1). L'insecte enserre une tige herbacée au moyen de ses mandibules contractées et de la première paire de pattes; les deux autres paires sont 


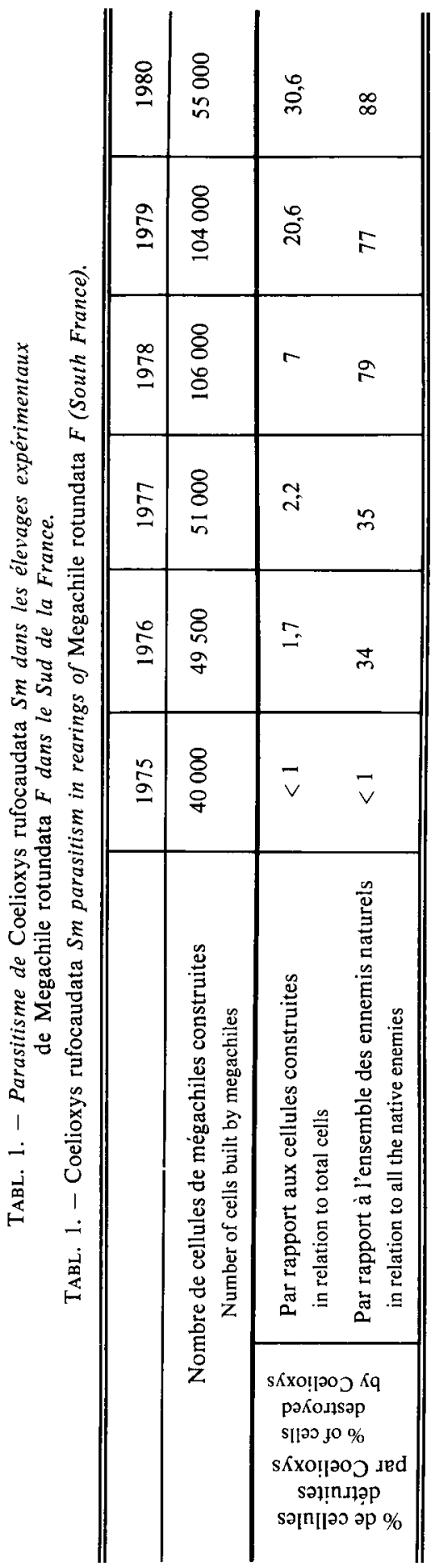




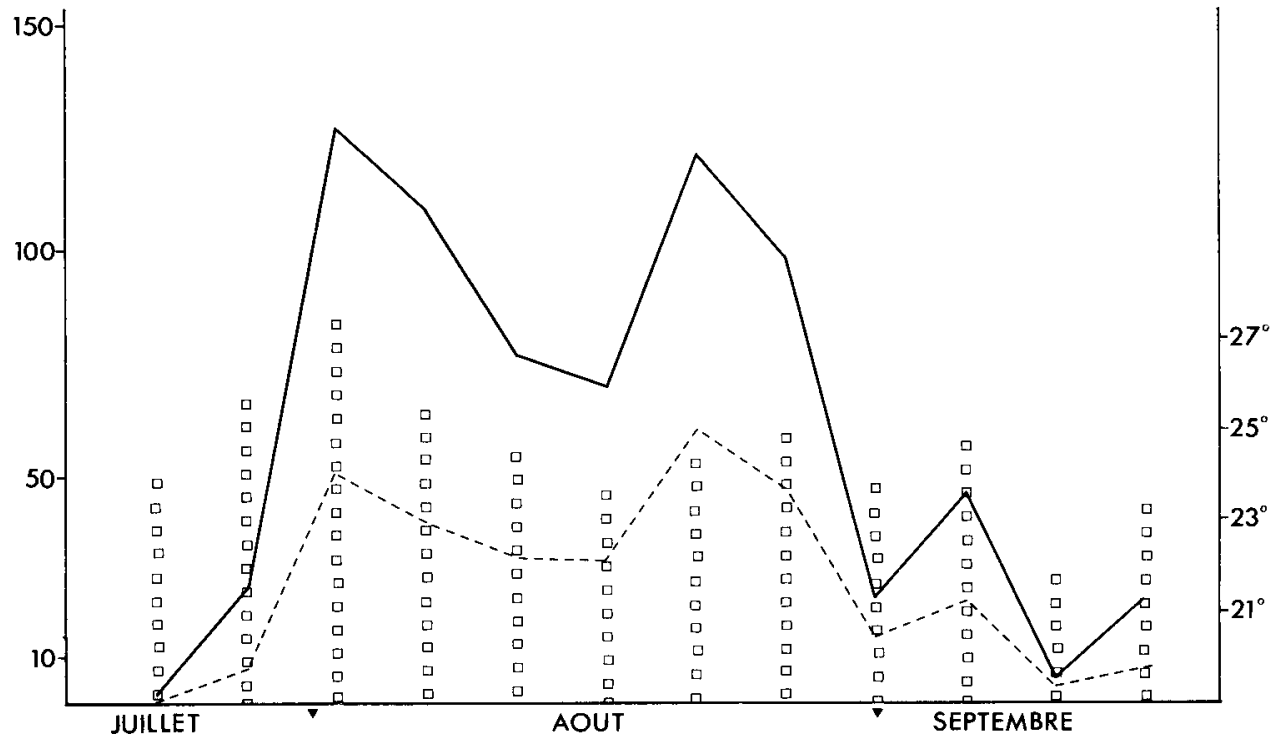

FIG. 1. - Parasitisme de Coelioxys rufocaudata au cours de la nidification de Mégachile rotundata en 1980. $--=$ cellules construites par $M$. rotundata; -.. = cellules parasitées par C. rufocaudata;

$\square \square \square=$ moyenne des températures maxima sur cinq jours.

Verticalement $=\mathrm{nb}$ de cellules (à gauche), température (à droite).

FIG. 1. - Parasitism of Coelioxys rufocaudata during Megachile rotundata flight in 1980.

$--=$ cells built by $M$. rotundata;

-. = cells parasitized by Coelioxys rufocaudata;

$\square \square \square=$ maximum average temperature on five days.

Vertically $=$ number of cells (left), temperature (right).

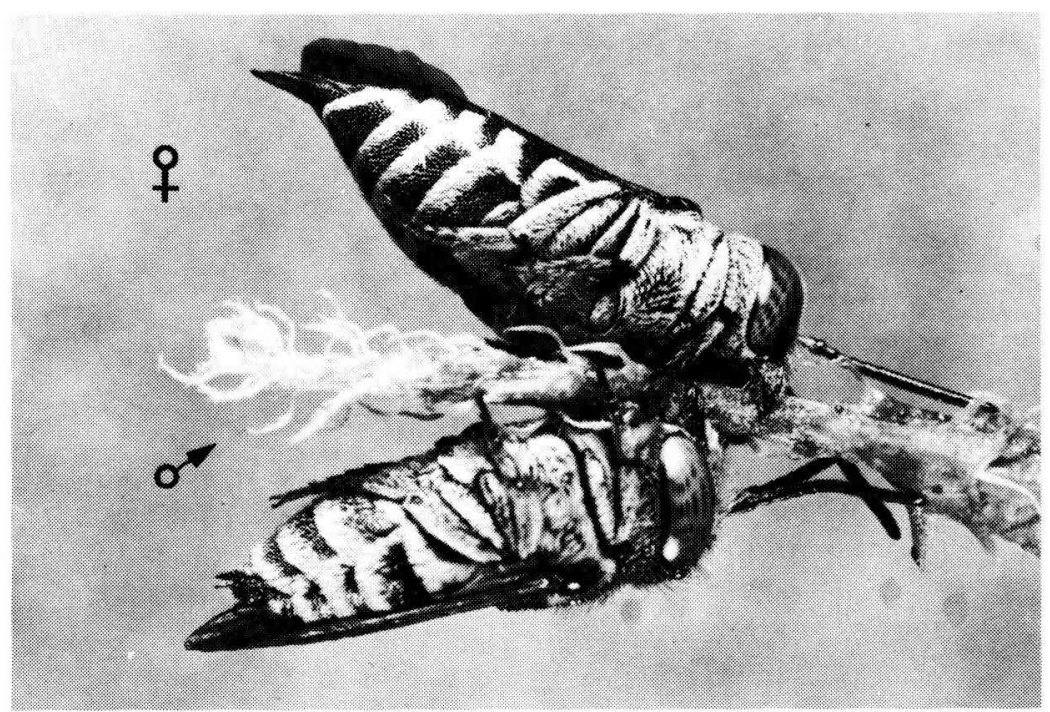

Рното 1. - Mâle et femelle de Coelioxys rufocaudata en position de sommeil sur une tige de mélilot. Photo 1. - Male and female of Coelioxys rufocaudata resting on Melilotus stem. 
repliées le long de l'abdomen, les antennes en extension dans le prolongement du corps. Cette position caractérise chez les deux sexes le repos nocturne. En serre celui-ci s'observe sur les extrémités des tiges ou des inflorescences de mélilot, les mâles et les femelles sont souvent groupés sur quelques centimètres de tige. Celle-ci peut servir de " perchoir " pendant plusieurs nuits successives, ce qui rend plausible l'existence d'un marquage olfactif. En conditions naturelles les aires de repos n'ont pu être localisées. Les phases de repérage, d'inspection des nids et d'alimentation sont entrecoupées de périodes de repos courtes ou d'attente sur les nichoirs ou leurs abords pendant lesquelles s'effectuent notamment toilettage et accouplement (1 à $2 \mathrm{mn}$ ). Pour s'alimenter les Coelioxys prélèvent le nectar des papilionacées proposées sans déclencher les fleurs. En conditions naturelles leur longévité peut atteindre plus de deux mois.

La séquence complète du comportement de ponte se déroule de la façon suivante : au vol de repérage en station fixe devant le nid succède un arrêt devant le tube choisi accompagné d'une pénétration de la tête à l'intérieur du nid avec battement alternatif des antennes ( 1 à $2 \mathrm{~s}$ ). L'inspection du nid peut alors se poursuivre et dure de 3 à $10 \mathrm{~s}$. Cette phase est suivie d'un retournement à l'entrée du nid ou à l'intérieur si le diamètre du tube est supérieur à $6 \mathrm{~mm}$. L'ovipositeur peut alors entrer en contact avec la cellule d'élevage. Cette phase est d'une durée beaucoup plus variable, de $11 \mathrm{~s}$ à $3 \mathrm{mn}$. Les tentatives de pénétration ne sont pas toujours suivies de la ponte comme l'attestent les sillons plus ou moins profonds laissés par l'organe de ponte sur la provision. Un deuxième retour rapide au nid, tête en avant, peut se produire après la ponte. L'œuf est situé dans sa presque totalité à l'extérieur de la cellule (Photo 2). Il peut être plus ou moins dissimulé par les morceaux de feuille les plus externes. La partie externe de l'œuf repose

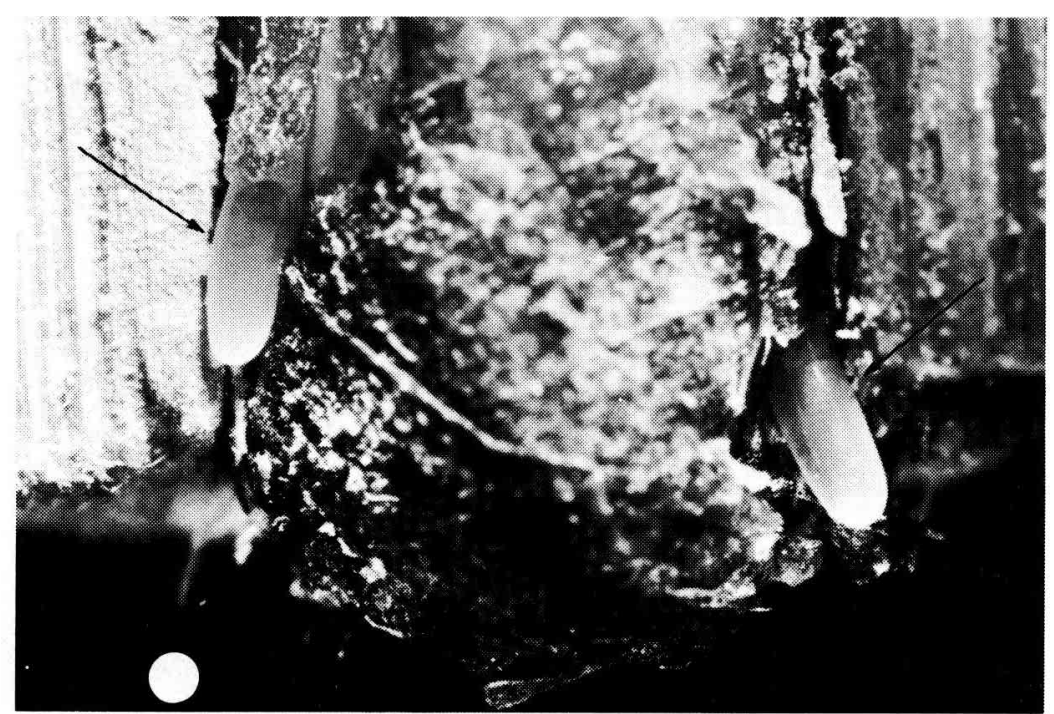

Pното 2. - Eufs de Coelioxys rufocaudata dans une cellule de Megachile rotundata. Pното 2. - Eggs of Coelioxys rufocaudata inserted in Megachile rotundata cell. 
dans une légère dépression créée par le retrait de la valve inférieure du dernier segment abdominal. Seule la partie céphalique de l'œuf est en contact avec la provision pollinique. Plusieurs femelles peuvent pondre dans la même cellule. Dans les conditions d'élevage en serre, le nombre d'œufs varie de 1 à 9 par cellule et de 1 à 4 en champ. La ponte intervient uniquement si le fond de la cellule est garni d'une couche de pollen de 2 à $3 \mathrm{~mm}$ d'épaisseur. La fréquence des pontes est maximum chez les cellules à moitié approvisionnées, elle diminue fortement en fin de remplissage et elle est nulle après la ponte du mégachile.

Dans un test préliminaire, des Coelioxys sont élevés sans leur hôte; des tubes de bambou garnis des cellules de mégachile en cours d'approvisionnement ont permis d'observer plusieurs pontes. La cellule de l'hôte à elle seule semble fournir les stimuli tactiles nécessaires à la ponte. Dans le cas d'un élevage en serre d'un autre Megachilidae caulicole (1) Osmia coerulescens Panz. en présence de Coelioxys, nous n'avons relevé aucune ponte bien que les nids aient été visités.

\section{Le développement larvaire - Morphologie et comportement.}

L'incubation dure environ 3 jours à $24^{\circ} \mathrm{C} \pm 1{ }^{\circ} \mathrm{C}$ et le développement des cinq stades larvaires jusqu'au filage du cocon de 11 à 16 jours.

Le troisième stade prédateur est atteint 6 à 7 jours après la ponte qui a toujours lieu avant celle de l'hôte alors que l'incubation de l'œuf de Megachile rotundata dure de 4 à 5 jours dans les mêmes conditions (MASURE, 1975). C'est donc le stade " œuf " très évolué ou la jeune larve de Megachile qui est dévorée par le parasite.

\section{L'Euf.}

L'œuf fraîchement pondu est translucide et de forme cylindrique. Il est légèrement arqué dorso-ventralement et présente un renflement à son pôle céphalique, immergé dans la masse pollinique. Sa longueur totale varie de 1,13 à $1,80 \mathrm{~mm}$, son diamètre dans la partie médiane entre 0,44 et $0,60 \mathrm{~mm}$.

Au-delà de 30 à 48 heures, la segmentation interne devient très visible, en fin d'incubation une série de mouvements de plus en plus rapides affecte la tête de l'embryon. Celui-ci absorbe peu à peu le liquide amniotique et environ 72 heures après la ponte le labre de la larve de $1^{\text {er }}$ stade est en contact avec le milieu semi-liquide de la provision.

\section{Premier stade larvaire.}

L'ouverture de la cellule et le prélèvement de l'individu sont indispensables pour la caractérisation de ce stade très fragile. En effet, la larve de premier stade ne déborde pas l'espace antérieurement occupé par le pôle apical de l'œuf tout en s'alimentant au contact de la provision. Les grains de pollen ingérés sont visibles au microscope. Cependant il n'y a pas encore de modification de la coloration de la partie externe. C'est le stade le plus court de la phase alimentaire ( 1 à 2 jours).

(1) Qui niche dans les tiges creuses. 
Plus large que longue, la capsule céphalique mesure $0,5 \mathrm{~mm}$ dans sa plus grande largeur. Les palpes maxillaires et labiaux ne sont pas visibles. Les ébauches antennaires sont de légères proéminences. L'absence de soies est totale. Les mandibules sont aiguës et transparentes. Elles sont munies d'une seule épine dorsale (Fig. 2). L'abdomen de la larve est arrondi à son extrémité et faiblement segmenté.

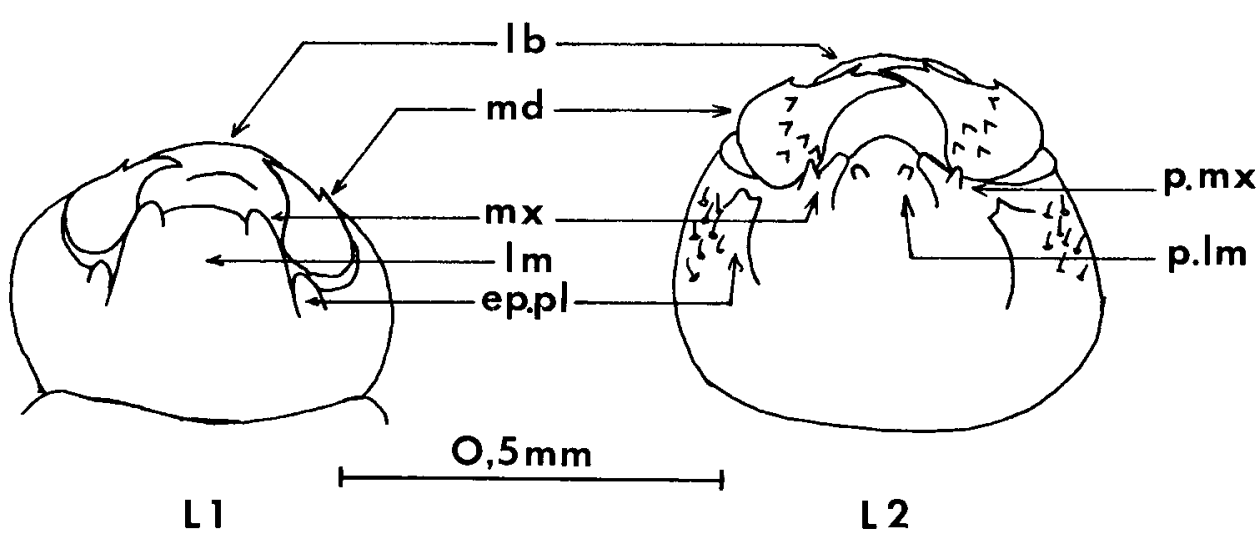

FiG. 2. - Capsule céphalique du premier et second stade larvaire de Coelioxys rufocaudata (vue ventrale).

$\mathrm{lb}=$ labre, $\mathrm{md}=$ mandibule, $\mathrm{mx}=$ maxille, $\mathrm{lm}=$ labium, ep. $\mathrm{pl}=$ épaississement du pleurostome,

p. $m x=$ palpe maxillaire, $\mathrm{p} . \operatorname{lm}=$ palpe labial, $\mathrm{a}=$ ébauche antennaire.

FIG. 2. - Coelioxys rufocaudata first and second instar head capsule (ventral view). $\mathrm{lb}=$ labrum, $\mathrm{md}=$ mandible, $\mathrm{mx}=$ maxilla, $\mathrm{lm}=$ labium,

ep. $\mathrm{pl}=$ pleurostomal thickening, $\mathrm{p} . \mathrm{mx}=\operatorname{maxillary}$ palp, $\mathrm{p} .1 \mathrm{~m}=$ labial palp, $\mathrm{a}=$ antenna.

\section{Le deuxième stade larvaire.}

Ce stade dure environ deux jours. La partie postérieure de la larve occupe toujours le chorion à l'extérieur de la cellule de Megachile alors que la tête a légèrement progressé à l'intérieur de la masse pollinique (Fig. 4). L'accroissement de la longueur du corps reste peu important. La segmentation est très évidente ainsi que la coloration jaunâtre de la colonne de pollen dans le tube digestif. Les stigmates sont très visibles, ceux qui sont situés dans la partie postérieure étant plus développés. La capsule céphalique (Fig. 2) large de 0,5 à $0,6 \mathrm{~mm}$ se caractérise par les éléments suivants : les mandibules ont une rangée d'épines à leur base et commencent à se sclérifier à leur extrémité. Les palpes maxillaires et labiaux sont bien individualisẻs ainsi que les épaississements du pleurostome. Les ébauches antennaires sont nettement différenciées. Le bord antérieur du labre est crénelé. Le pleurostome et la région labro-clypéale présentent de nombreuses soies.

Le troisième stade larvaire.

Ce stade qui émerge de la provision caractérise la phase prédatrice du parasite. La longueur des mandibules le différencie des autres stades. En outre, l'existence d'un 

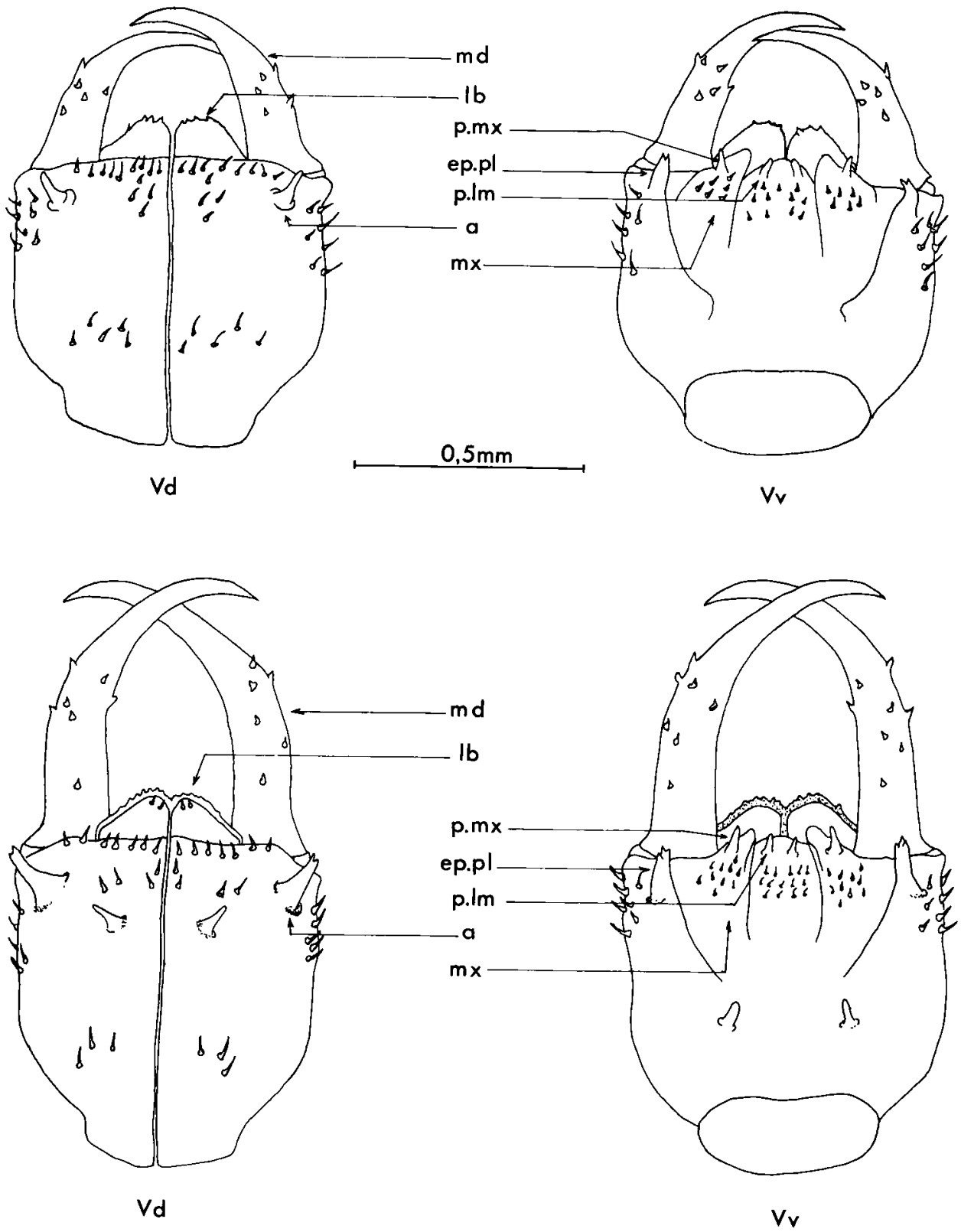

FIG. 3. - Capsules céphaliques du troisième stade larvaire (mâle et femelle) de Coelioxys rufocaudata.

$\mathrm{lb}=$ labre, $\mathrm{md}=$ mandibule, $\mathrm{p} . \mathrm{mx}=$ palpe maxillaire, $\mathrm{p} . \mathrm{lm}=$ palpe labial, ep. $\mathrm{pl}=$ épaississement du pleurostome, $\mathbf{a}=$ ébauche antennaire, $\mathrm{mx}=$ maxille, $\mathrm{Vd}=$ vue dorsale, $\mathrm{Vv}=$ vue ventrale.

FIG. 3. - Male and female Coelioxys rufocaudata head capsules (third instar).

Ib = labrum, $\mathrm{md}=$ mandibule, $\mathrm{p} . \mathrm{mx}=$ maxillary palp, $\mathrm{P} . \mathrm{lm}=$ labial palp, ep. pl = pleurostomal thickening, $a=$ antenna, $m x=$ maxilla, $\mathrm{Vd}=$ dorsal view, $\mathrm{Vv}=$ ventral view. 
dimorphisme sexuel mérite que l'on s'y attarde davantage. Au cours de ce stade d'une durée de 4 à 6 jours, la larve va utiliser le chorion comme point d'ancrage des derniers segments abdominaux pour progresser en extension vers la surface (Fig. 4). Elle le quittera ensuite pour reprendre une position plus arquée après avoir atteint la surface. Le

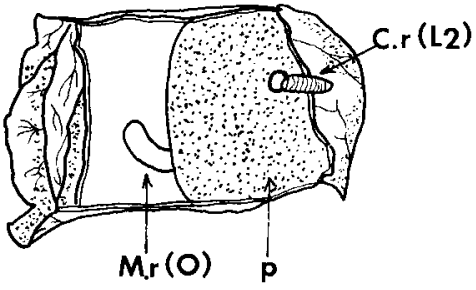

1

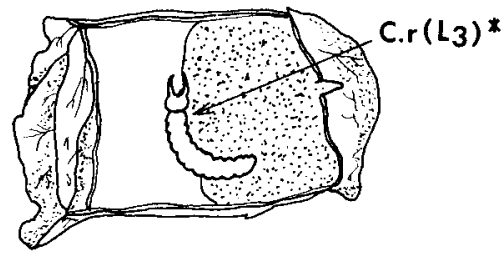

3

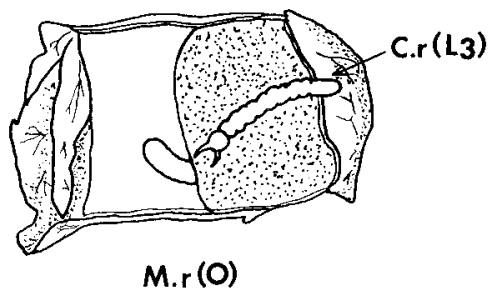

2

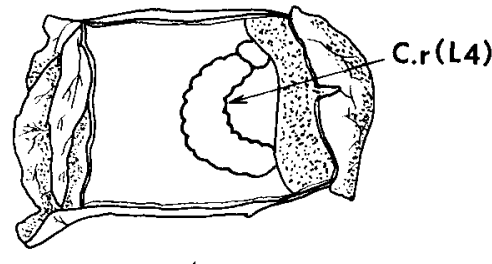

4

FIG. 4. - Evolution des stades larvaires $L 2, L 3, L 4$ de Coelioxys rufocaudata. $\mathrm{Mr}(\mathrm{o})=$ œuf de Megachile rotundata, $\mathrm{C} . \mathrm{r}=$ Coelioxys rufocaudata,

$(\mathrm{L} \mathrm{3})^{*}=$ troisième stade larvaire après destruction de l'hôte, $\mathrm{p}=$ pollen.

FIG. 4. - Evolution of Coelioxys rufocaudata in host cell ( 2 nd 3 rd and 4 th instar). $\mathrm{Mr}$ (o) M. rotundata egg, c.r. = C. rufocaudata, (L 3)* third instar after host destruction, $\mathrm{p}=$ pollen.

comportement agressif à l'égard de l'hôte se manifeste avant même l'émergence totale de la capsule céphalique : les mandibules s'ouvrent et se ferment dans toutes les directions à la surface des réserves, ce qui entraîne généralement la mort de l'œuf ou de la jeune larve de mégachile qui est vidée de sa substance en quelques heures. La larve poursuit ensuite son alimentation sur la provision. Malgré le nombre élevé d'œufs rencontrés parfois sur des cellules il ne subsiste toujours qu'une seule larve en fin de $3^{\mathrm{e}}$ stade. L'existence de 1 à 2 capsules de L 3 supplémentaires à la surface du cocon après filage implique une forte compétition intraspécifique où le dimorphisme sexuel peut jouer un rôle important. La capsule céphalique très sclérifiée est fréquemment retrouvée intacte au milieu des excréments rejetés par la larve au cours du filage du cocon. Les mandibules sont très longues et acérées avec de nombreuses épines sur leur partie basale (Fig. 3). Celles du mâle sont nettement plus courtes ( $L=0,443 \pm 0,015 \mathrm{~mm}$ ) que la largeur de la capsule céphalique $(\mathrm{L}=0,657 \pm 0,015 \mathrm{~mm})$. Celles de la femelle atteignent $0,771 \pm 0,038 \mathrm{~mm}$ soit au moins la largeur de la capsule céphalique $(\mathrm{L}=0,744 \pm 0,030 \mathrm{~mm})$. Chez les deux sexes les palpes maxillaires et labiaux ont leur taille maximale ainsi que les ébauches antennaires et les épaississements du pleurostome. Les maxilles et le labium présentent une forte pilosité. 
Quatrième stade larvaire.

Ce stade d'une durée de 2 à 3 jours est marqué par le retour à une morphologie plus spécifique des larves de mégachilidés. Les mandibules de la phase prédatrice ont disparu, et la larve s'alimente exclusivement sur la provision qu'elle dévore en grande partie (Fig. 4). L'accroissement de taille est rapide et il y a rejet des premières pelotes fécales. La coloration plus foncée et plus hétérogène de l'abdomen de la larve de Coelioxys permet sa distinction rapide de la larve de mégachile d'un blanc nacré.

\section{Cinquième stade larvaire.}

Sur le plan morphologique, il y a régression totale des excroissances pleurostomales (Fig. 4) et apparition d'une pilosité abondante sur les segments abdominaux.

Pendant 2 à 3 jours, la larve de cinquième stade achève les provisions polliniques et rejette ses excréments au sommet de la cellule qu'elle commence à tapisser de filaments soyeux, blancs au début et devenant rougeâtres par la suite. Le cocon terminé se distingue généralement du cocon de $M$. rotundata par son extrémité antérieure subsphérique et souvent en retrait par rapport à l'opercule de feuilles alors que celui de $M$. rotundata est plat et adhère à l'opercule. Le tissage de cette même partie est plus grossier chez $C$. rufocaudata avec de nombreux filaments rougeâtres en structure lâche. La confirmation de l'identification est presque toujours apportée par la présence d'une ou plusieurs capsules céphaliques de $3^{\mathrm{e}}$ stade et quelquefois de $4^{\mathrm{e}}$ stade parmi les pelotes fécales (Photo 3). Après filage du cocon la larve entre en diapause et passe l'hiver à l'état de prénymphe, comme son hôte.

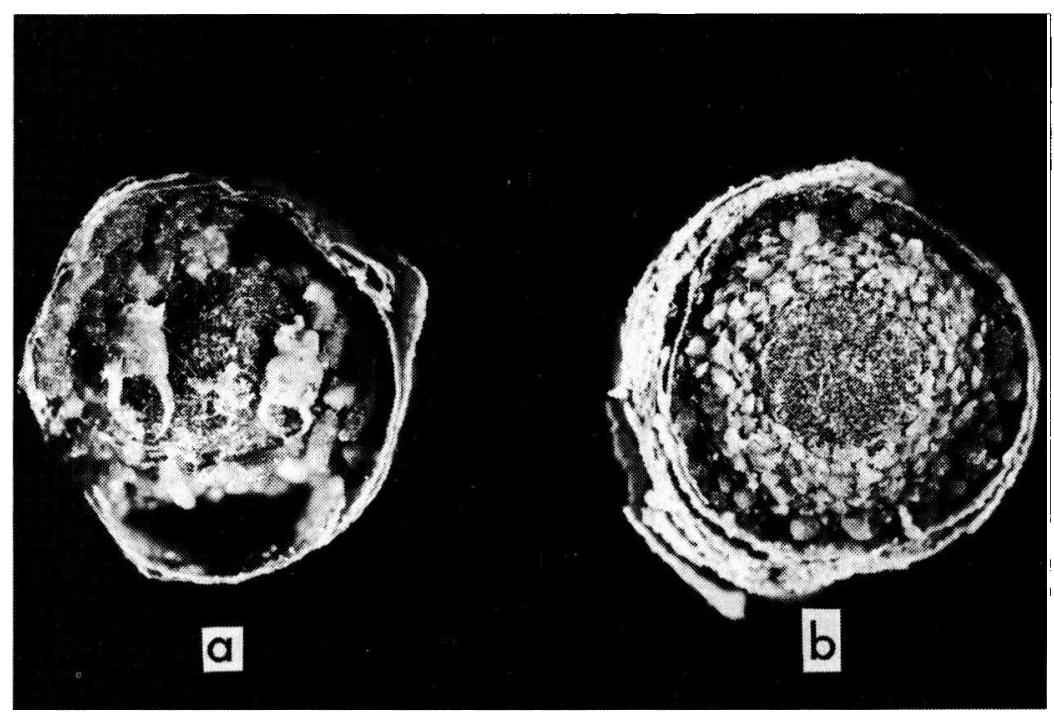

Pното 3. - a) Pôle apical d'un cocon de Coelioxys rufocaudata avec 2 capsules céphaliques de troisième stade.

b) Pôle apical d'un cocon de Megachile rotundata.

Pното 3. - a) Apical part of Coelioxys rufocaudata cocon with two third instar head capsules. b) apical part of Megachile rotundata cocoon. 


\section{DISCUSSION ET CONCLUSION}

La difficulté d'élever et d'observer le développement post-embryonnaire des larves d'" abeilles coucous " explique les contradictions relevées dans la littérature au sujet du nombre de stades larvaires et de leur comportement. Par exemple, les premier et second stades larvaires sont ignorés par GRAENiCHER (1905 et 1927) et MiCHENER (1953 b). L'existence de cinq stades larvaires chez $C$. rufocaudata confirme les travaux de BAKer, 1972 sur C. octodentata SAY et $C$. sayi et de WAFA (1973) sur C. decipiens SPIN. Chez $C$. rufocaudata, les deux premiers stades restent fixés au chorion et s'alimentent au sein de la provision pollinique.

Bien que le second stade soit déjà pourvu de fortes mandibules, il semble que leur action ne s'exerce qu'aux dépens des larves concurrentes et non à celles de l'hôte. Le chorion sert encore d'appui au troisième stade pour atteindre la surface de la provision, l'extrémité de l'abdomen remontant ensuite progressivement. Un autre mode de locomotion se rencontre chez Triepeolus remigatus (Anthophoridae, Nomadinae) : la larve primaire est pourvue d'importantes expansions ventro-latérales (STEPHEN, 1964). L'existence d'un dimorphisme sexuel également relevé par BAKER peut constituer un avantage sélectif favorisant les femelles dans le cas de pontes multiples.

Le comportement de sommeil sur des tiges herbacées se rencontre aussi avec des variations chez des Anthophoridae : Anthophora (STEPHEN et al., 1969), Holcopasites (RozEN, 1965), Triepeolus (BoHART, 1970) ainsi que chez un autre Megachilidae: Anthidiellum (STEPHEN, 1969).

Le parasitisme de Megachile rotundata par l'espèce paléarctique C. rufocaudata $S m$ est signalée en France par TASEI (1975), mais également en Espagne par AsCENSIO (1972) et en Hongrie par MANNiNGER (1972) qui n'en chiffrent pas l'importance. Aux États-Unis, Waters, en 1971, estime que Coelioxys sp. est un parasite d'ordre secondaire et PANKIW (1977) mentionne des pertes inférieures à $1 \%$ alors que le taux maximum de cellules parasitées par C. rufocaudata atteint 44,5\% dans l'un de nos élevages et $30,6 \%$ en moyenne en 1980. Cette progression semble avoir été limitée en 1980 par le retrait précoce des éclosoirs qui n'ont libéré ainsi qu'une fraction de la population de Coelioxys. D'après la littérature, il semble que les espèces de Coelioxys identifiées dans les élevages américains soient plutôt spécifiques de mégachiles locaux (MEDLER et KOERBER, 1948) occupants secondaires des nichoirs à Megachile rotundata: leur émergence plus précoce que celle de l'hôte domestiquée permet leur élimination au moment de l'incubation. A l'opposé, C. rufocaudata Sm. parasite spécifique de $M$. rotundata univoltin est particulièrement adapté au cycle biologique et au comportement de son hôte.

Son taux de multiplication, voisin de 3 pour deux années consécutives, menace d'extinction notre souche canadienne de mégachiles. Son introduction sur le continent 
américain aurait des suites fâcheuses. Dans la perspective d'une méthode de lutte, on envisage d'étudier le synchronisme des émergences du parasite et de son hôte ainsi que les caractères de la phase de repos nocturne.

Reçu pour publication en juin 1981 .

Eingegangen im Juni 1981 .

\title{
ZUSAMMENFASSUNG
}

\begin{abstract}
COELIOXYS RUFOCAUDATA SM. (HYM., MEGACHILIDAE) EIN KLEPTOPARASIT VON MEGACHILE ROTUNDATA F. (HYM., MEGACHILIDAE, EINEM BESTÄUBER DER LUZERNE.
\end{abstract}

Der Parasitismus, der die künstlichen Zuchten der luzernebestäubenden Megachile rotundata in Mitleidenschaft zieht, kann deren Vermehrung begrenzen. Im Jahre 1980 lagen die Verluste, die von Coelioxys rufocaudata Sm. (Megachilidae), einer " Kuckucksbiene " verursacht wurden, zwischen 30 und 44,5\%. Das hat die Autoren veranlasst, Art und Weise des Befalls und die Biologie des Kleptoparasiten zu untersuchen. Denn die Kenntnisse über ihn sind nur gering und oft widersprüchlich, besonders hinsichtlich der Larvenentwicklung.

Die Resultate beruhen auf folgenden Untersuchungen :

- Experimentelle Aufzucht der Megachile im Süden Frankreichs (Tab. I).

- Aufzucht des Wirts und seines Parasiten auf einem Luzernefeld in Lusignan (Abb. 1).

- Aufzucht beider Arten im Glashaus auf Melilotus und 2 Nistplätzen aus Bambus-Stengeln.

- Aufzucht von Eiern und Larven von Coelioxys rufocaudata im Brutschrank bei $24 \pm 1^{\circ} \mathrm{C}$ und einer relativen Luftfeuchte von $60 \%$.

Während der Aufzucht auf einem Luzernefeld in Lusignan im Jahr 1980 war Coelioxys vom 17. Juli bis zum 22. September aktiv (Abb. 1). Es gab keine zweite Generation. 1980 lag der Anteil der Weibchen zwischen 27,8 und 48,8\%. Die allgemeine Aktivität und das Eilegeverhalten wurden ebenfalls studiert. Mehrere Weibchen können in dieselbe Zelle legen (1-4 Eier). Das Weibchen durchbohrt vor der Eiablage die Pollenvorräte und die Blatthülle. Der rückwärtige Teil des Eies ragt aus der Zelle nach aussen hervor (Photo 2) und der kephale Pol steht mit dem Pollen in Berührung. Um die Eiablage auszulösen, ist eine Pollenschicht von 2-3 mm Dicke erforderlich. Sobald die Zellen fertig versorgt sind, werden keine Eier mehr abgelegt. Die Inkubationszeit bis zum Schlüpfen der Larve dauert bei $24^{\circ} \mathrm{C}$ drei Tage. Die Entwicklung bis zum Spinnen des Kokons durchläuft fünf Larvenstadien und dauert 11-16 Tage. Die beiden ersten Stadien (Abb. 3) bleiben mit dem Chorion in Verbindung und bewegen sich geringfügig in die Mitte der Pollenmasse. 6-7 Tage nach dem Ausschlüpfen verlässt die Larve des dritten Stadiums das Chorion und taucht an der Oberfläche auf (Abb. 4). Ihre prognathe Kopfkapsel ist mir sehr starken Mandibeln ausgestattet, die beim Weibchen länger sind (Abb. 3). Diese Larve frisst den Wirt im Stadium der fortgeschrittenen Eientwicklung oder der jungen Larve. Das 4. und das 5. Larvenstadium zeigen eine stärker für Megachilidae spezifische Morphologie mit starker Anpassung an eine Pollenernährung. Am Ende des fünften Stadiums erfolgt das Spinnen des Kokons. Das Vorhandensein einer Kopfkapsel des 3. Stadiums, die aus dem Kokon herausragt und die gröbere Textur der apicalen Partie des Kokons gestatten im allgemeinen die Unterscheidung der Zelle des Parasiten von der seines Wirtes (Photo 3). Die Diapause erfolgt im praenymphalen Stadium wie bei dem Wirt.

Unsere Ergebnisse bestätigen die Arbeiten von BAKER (1971), der als erster das Vorhandensein von fünf Larvenstadien bei Coelioxys octodentata SAY und Coelioxys sayi nachgewiesen hat. BAKER hat auch einen sexuellen Dimorphismus bei der Larve des 3. Stadiums beschrieben. Aus Spanien und aus Ungarn berichten ASENSIo (1972) und MANNINGER (1972) das Vorkommen von Coelioxys rufocaudata Sm. unter den natürlichen Feinden von Megachile rotundata F. PANKIW und LIEvERSE (1977) schätzen die Höhe der durch Coelioxys sp. verursachten Verluste auf unter $1 \%$ und halten sie für die kanadischen Zuchten nicht für bedrohlich. Die Einschleppung der palaearktischen Art Coelioxys rufocaudata nach Nordamerika könnte für die amerikanischen Populationen der Blattschneidebienen schlimme Folgen haben. In Hinblick auf eine Bekämpfungsmethode wird eine Vertiefung der Untersuchung über die Verzögerung des Ausschlüpfens des Parasiten und seines Wirtes und der nächtlichen Ruhephase geplant. 


\section{SUMMARY \\ COELIOXYS RUFOCAUDATA SM (HYM., MEGACHILIDAE) CLEPTOPARASITE OF THE ALFAFA LEAFCUTTER BEE (MEGACHILE ROTUNDATA F.)}

Parasitism affecting experimental rearings of the alfalfa leafcutter bee, Megachile rotundata $F$., may often prevent population increase. In 1980 losses due to Coelioxys rufocaudata Sm (Megachilidae) varied between 30 and $44,5 \%$. This led the authors to study the process of infestation and the biology of this cleptoparasite on which scientific knowledge is scarce and inconsistent, especially in the field of larval development.

Our results come from:

- experimental rearings of leafcutter bees in southern France (Table 1);

- rearing of the host and its parasite in Lusignan area within an alfalfa field in 1980 (Fig. 1);

- rearings of the two species in greenhouse supplied with Melilotus bloom and bamboo trapnests;

- rearings of eggs and larvae of Coelioxys rufocaudata $\mathrm{Sm}$ in incubator at $24^{\circ} \pm 1{ }^{\circ} \mathrm{C}$ with $60 \%$ relative humidity.

In the alfalfa field at Lusignan, Coelioxys were active from 17 July to 22 September. Several females were observed to lay eggs in the same cell (one to four eggs). The female perforates pollen mass and leaf lining to lay an egg. The posterior end of this egg protudes out of the cell (Photo 2) and the cephalic end touches the pollen. Coelioxys females start egg laying when pollen provisions reach 2 to $3 \mathrm{~mm}$ but don't deposit in completed cells. At $24^{\circ} \mathrm{C}$ egg incubation lasts three days. Before cocoon spinning, five larval stages occur in 11 to 16 days. The first two stadia (Fig. 2), still partly in the egg chorion, move slightly forward to the middle of pollen mass (Fig. 4). Six to seven days after hatching, the third stage leaves the chorion and crawls to the surface of the provision (Fig. 4). The prognathous head capsule of this stadium has very strong mandibles and is longer in the female (Fig. 3). The parasite larva consumes the host egg or young larva. The morphology of the fourth and fifth stage is more resemblant of other Megachilidae and better adapted for consuming the host provisions. Cocoon spinning takes place during the fifth stage. The presence of cephalic capsule of the third stage lies outside of the cocoon and the rough texture of the apical end of cocoon characterize Coelioxys (Photo 3). The diapausing stage is prepupae as in the host. No second generation was observed.

Our results agree with BAKER (1971), who was first to demonstrate the existence of five larval stages in Coelioxys octodentata Say and Coelioxys sayi. BAKER also reported a sexual dimorphism in the third larval stage. In Spain and in Hungary, AsENsio (1972) and MANNINGER (1972) observed the existence of Coelioxys rufocaudata among native enemies of Megachile rotundata. PANKIW and LiEverse (1977) estimated that losses due to Coelioxys sp. are lower than $1 \%$ and don't affect Canadian rearings.

The introduction of the palearctic species Coelioxys rufocaudata in the New World would certainly cause great damage to the American leafcutter bee populations.

The study of emergence patterns and nocturnal rest periods of both host and parasite will be of interest for finding a control method.

\section{BIBLIOGRAPHIE}

Asensio de LA SIERRA E., Rodriguez Ibanez J. A., 1972. - Primer informe sobre adaptacion y multiplicacion de lá abeja polinizadora de la alfalfa Megachile pacifica Panz (M. rotundata auct. nec. Fabricius). An. Inst. Nac. Invest. Agrar. Ser. Prot. veg., 2, 237-252.

BAKer J. R., 1971. - Development and sexual dimorphism of larvae of the bee genus Coelioxys. Kans. Entomol. Soc., 44, 2, 225-235.

BoharT G. E., 1966. - Notes on Triepeolus remigatus Fab, a " cuckoo bee " parasite of the squash bee, Xenoglossa strenva Cresson (Hymenoptera : Apoidea). Pan. Pac. Entomol., 42, 255-262. 
Ferton Ch., 1896. - Nouvelles observations sur l'instinct des Hyménoptères gastrilégides de la Provence. Actes Soc. Linn. Bordeaux, 48, 241-249.

FrIESE H., 1923. - Die europäischen Bienen (Apidae). Walter de Grayter and Co, Berlin and Leipzig.

GraENicher S., 1905. - Some observations on the life history and habits of parasitic bees. Bull. Wiscon$\sin$ Natur. Hist. Soc., 3, 153-167.

Graenicher S., 1927. - On the biology of the parasitic bees of the genus Coelioxys (Hymen, Megachilidae). Entomol. News, 38, 231-235, 273-276.

Hardouin R., 1948. - La vie des abeilles solitaires. Hist. nat. 9, Gallimard.

IWATA K., 1939. - Biology of Coelioxys elongata lepeletier. Mushi, 12, 34-40.

MANninger S., 1972. - Parasites of alfalfa leaf-cutter bee, Megachile rotundata F. (M. pacifica Panz) in Hungary. Novenytermeles, 21, 321-328.

MASURE M. M., 1975. - Étude de quelques phénomènes relatifs à la diapause d'une abeille solitaire pollinisatrice de la luzerne Megachile pacifica Panz. (donnée non publiée).

Medler J. T., Koerber T. W., 1958. - Biology of Megachile relativa Cresson (Hymenoptera, Megachilidae) in the trap-nests in Wisconsin. Ann. Ent. Soc. America, 51, 337-344.

Michener C. D., 1953 a. - The biology of a leafcutter bee (Megachile brevis) and its associates. Univ. Kansas Sci. Bull., 35, 1659-1748.

MiCHENER C. D., 1953 b. - Comparative morphological and systematic studies of bee larvae with a key to the families of hymenopterous larvae. Univ. Kansas Sci. Bull., 35, 987-1102.

Pankiw P., Lieverse J. A. C., 1977. - The alfalfa leafcutter bee (Megachile pacifica) in Northwestern Canada. Contribution $n^{\circ}$ NRG 77-2.

Perez J., 1889. Les abeilles. - Paris Hachette, 1889.

Bohart G. E., 1970. - The evolution of parasitism among bees. St. Faculty Honor. Lecture Utah State Univ., vol. 41, 1-30.

Richards O. W., 1949. - The evolution of cuckoo bees and wasps. Proc. Linnean Soc. London, 161, 4041.

Rozen J. G., 1965. - Biological notes on the cuckoo bee genera Holcopasites and Neolarra (Hymenoptera : Apoidea). New-York Entomol. Soc., vol. 73, 87-91.

Rozen J. G., Michener C. D., 1968. - The biology of Scrapter and its Cuckoo bee Pseudodichroa (Hymenoptera : Colletidae and Anthophoridae). Amer. Mus. nov. $\mathrm{n}^{\circ} 2335,1-13$.

ROzEN J. G., 1969. - The biology and description of a new species of African Thyreus with life history notes on two species of Anthophora (Hymenoptera : Anthophoridae). J. New York Entomol. Soc., 77, $51-60$.

StePhen W. P., Bohart G. E., Torchio P. F., 1969. - The biology and external morphology of bees with a synopsis of the genera of Northwestern America. Agr. Exp. Sta. Oregon State Univ., Corvallis, $140 \mathrm{p}$.

TASEI J. N., 1972. - Observations préliminaires sur la biologie d'Osmia (Chalcosmia) coerulescens $L$. (Hymenoptera megachilidae), pollinisatrice de la luzerne (Medicago sativa L.). Apidologie, 3, 149165.

TASEI J. N., 1975. - Le problème de l'adaptation de Megachile (Eutricharaea) pacifica Panz. (Megachilidae) américain en France. Apidologie, 6, 1-57.

WAFA A. K., MAZEed M. M., 1973. - Natural ennemies of pollinators belonging to family Megachilidae. Agr. Res. Rev. Cairo, 51, 1, 129-137.

WATERS N. D., 1971. - Insect enemies of the alfalfa leafcutter bee and their control. Idaho Current Inf. Series N. 163. 